\title{
Epithelioid Sarcoma Presenting as Recurrent Thumb Ulcer: A Lesson to
} Learn

\begin{abstract}
Epithelioid sarcoma (ES) first described by Enzinger in $1970,{ }^{[1]}$ is a rare soft-tissue sarcoma typically presenting as a subcutaneous or deep dermal mass in distal portions of the extremities of adolescents and young adults. They are frequently mistaken for ulcers, abscesses, or infected warts that fail medical management. Patients often develop multiple local recurrences of long duration, with subsequent metastases in $30 \%-50 \%$ of cases. ${ }^{[2]}$ We here report a case of left thumb ES that presented as an ulcer and subsequently metastasized to the forearm, arm, axillary lymph nodes, and lungs.

Keywords: Distal type sarcoma, epithelioid sarcoma, hand sarcoma, thumb ulcer
\end{abstract}

\section{Introduction}

Epithelioid sarcoma (ES) is a rare slow-growing soft-tissue sarcoma typically involving the distal extremity of young adults, with a strong propensity for local recurrence and ultimately metastasis. Microscopically, most tumors are characterized by a granuloma-like pattern: nodules of spindle and epithelioid cells circumscribe areas of central hyalinization and necrosis. In 1997, Guillou et al. described a different type of ES, called proximal-type ES, which is found mostly in the pelvic and perineal regions and genital tracts of young to middle-aged adults, characterized by a proliferation of epithelioid-like cells with rhabdoid features and absence of a granuloma-like pattern. ${ }^{[3]}$ These have a worse prognosis than those arising in distal locations.

\section{Case Report}

A 32-year-old male presented with complaints of swelling in his left thumb for 2 years duration. The swelling was nontender and progressively increased in size to involve the distal portion of the left thumb. The swelling underwent spontaneous rupture to form an ulcer 2 months before his hospital visit. The ulcer persisted despite multiple debridements and administration of several antibiotics. In November 2015, he underwent excision of the left thumb.

This is an open access journal, and articles are distributed under the terms of the Creative Commons Attribution-NonCommercial-ShareAlike 4.0 License, which allows others to remix, tweak, and build upon the work non-commercially, as long as appropriate credit is given and the new creations are licensed under the identical terms.

For reprints contact: reprints@medknow.com
Histopathological examination revealed a spindle cell neoplasm with epithelioid features. It also showed underlying bone and marrow infiltration by the tumor cells. Immunohistochemistry (IHC) shows diffuse positivity for epithelial membrane antigen and $\mathrm{CD} 34$ and focal positivity for cytokeratin and smooth muscle actin. Neoplastic cells were negative for S100. Ki-67 was around 25\% [Figure 1].

The patient had not received further treatment following amputation.

In February 2016, he presented with complaint of multiple, hard mobile swellings in his left forearm and arm, with enlarged left axillary lymph nodes [Figure 2]. Excisional biopsy of one of the forearm nodules confirmed a recurrence with the same histology with positivity for same IHC markers as seen in left thumb-amputated specimen. Computed tomography $(\mathrm{CT})$ thorax revealed multiple pulmonary metastases. left axilla showed an enlarged lymph node of size $4.2 \times 4 \mathrm{~cm}$.

The patient was started on chemotherapy consisting of doxorubicin and ifosfamide. Reassessment CT scan performed after 3 cycles of chemotherapy showed progressive disease. Second-line chemotherapy (gemcitabine and docetaxel) was started but the patient did not tolerate well and hence best supportive care was offered.

\footnotetext{
How to cite this article: Rajeev LK, Asati V, Mallekavu SB, Babu KG, Champka G. Epithelioid sarcoma presenting as recurrent thumb ulcer: A lesson to learn. Indian J Med Paediatr Oncol 2018;39:527-9.
}

\section{Lakkavalli \\ Krishnappa Rajeev, Vikas Asati, Suresh Babu Mallekavu, K Govind Babu, Champka G}

Department of Medical Oncology, Kidwai Memorial Institute of Oncology, Bengaluru, Karnataka, India

Address for correspondence: Dr. Vikas Asati, Department of Medical Oncology, Kidwai Memorial Institute of Oncology, Bengaluru, Karnataka, India. E-mail:vikas1asati@gmail.com

Access this article online

Website: www.ijmpo.org

DOI: 10.4103/ijmpo.ijmpo_87_17 Quick Response Code:

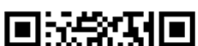

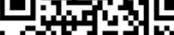
P4t87.

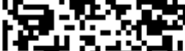
a :ping 


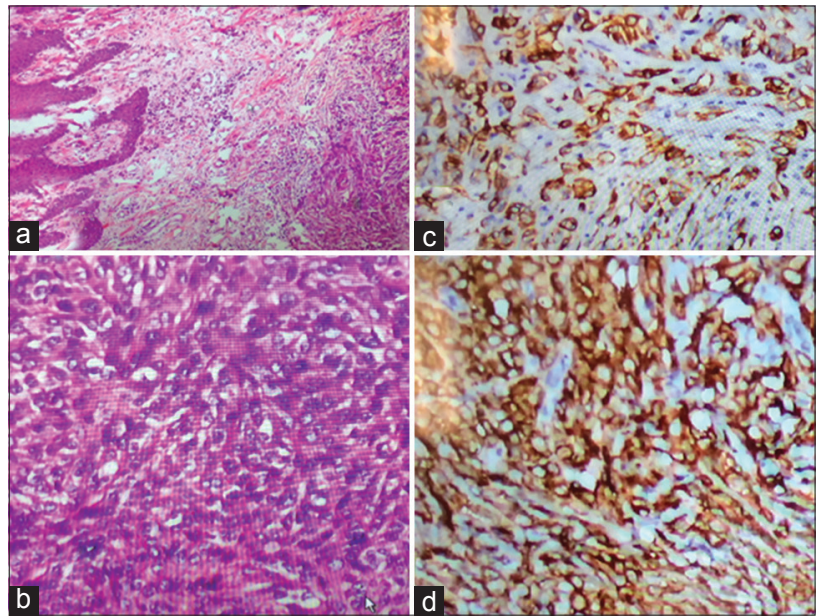

Figure 1: (a) Tumor involving the reticular dermis and subcutaneous tissue $(\mathrm{H}$ and $\mathrm{E}, \times 10)$. (b) Tumor cells are predominantly epithelioid and spindle shaped $(\mathrm{H}$ and $\mathrm{E}, \times 40)$. (c) Immunohistochemistry, tumor cells are cytokeratin positive. (d) Tumor cells are strongly positive for epithelial membrane antigen

\section{Discussion}

ES is a rare high-grade malignancy that represents the most common primary soft-tissue sarcoma of the hand. Because of its epithelial and mesenchymal differentiation, this tumor is often mistaken for chronic inflammatory conditions, necrotizing granulomas, and various fibrohistiocytic tumors. ${ }^{[4]}$ ES often presents in young men, but its significance is great because of an inherent propensity for local recurrence in addition to regional lymph node involvement and distant metastases. ${ }^{[5]}$ Males are disproportionately affected with a ratio approaching 2:1. ES initially appears as a single nodule. However, at the time of diagnosis, multiple nodules representing local disease spread may be present ${ }^{[6]}$ as seen in our patient. Periosteal bone invasion can occur. ${ }^{[1]}$

Radical tumor excision is the primary treatment for patients with ES. Negative tumor margins must be obtained, but optimal function, particularly of the upper limb, is also to be considered ${ }^{[7]}$ Therapeutic lymph node dissection is indicated when lymph node metastases are present. A decreased risk of local recurrence has been reported with the addition of preoperative or postoperative local radiation therapy when combined with radical surgery. ${ }^{[6]}$ Radiation can also be considered for patients with marginal primary resection, local regional recurrence, or palliative treatment.

Single agent or combination chemotherapy is the treatment offered in patients with Stage IV disease, as in our patient with lung metastasis. The most commonly administered chemotherapy regimens are single-agent anthracycline therapy or the combination of anthracycline with ifosfamide. ${ }^{[8]}$ A single group reported activity of a regimen combining gemcitabine with docetaxel, but the experience is limited to a small number of patients. ${ }^{[9]}$

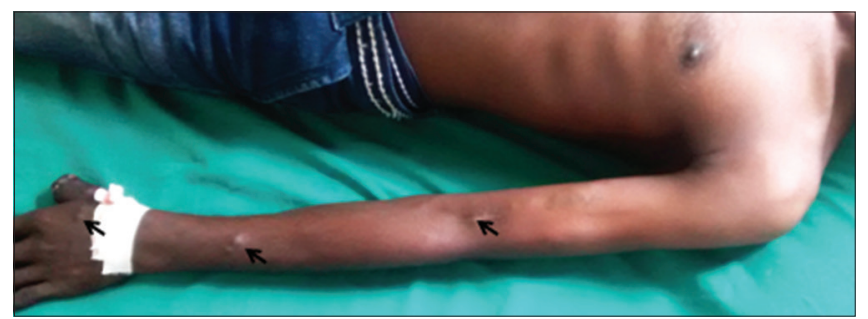

Figure 2: Clinical picture of the patient showing the partially amputated thumb and multiple cutaneous metastatic nodules over hand, forearm, and arm (arrows)

Despite the administration of palliative chemotherapy, patients with metastasis have poor prognosis. The reported median survival is around 52 weeks and the 1 and 5 year survival rates are $46 \%$ and $0 \%$, respectively. ${ }^{[8]}$ Therefore, its an unmet need to improve the medical management of ES patients by introducing novel agents.

Male gender, depth of invasion, and vascular invasion are associated with poor prognosis. Anatomic location of ES appears to play a role in prognostication. The overall survival and metastases-free survival are worse in lesions proximal to the elbow or knee. ${ }^{[10]}$ Close, long-term follow-up is necessary because recurrence and metastases may occur long after definitive treatment.

\section{Financial support and sponsorship}

Nil.

\section{Conflicts of interest}

There are no conflicts of interest.

\section{References}

1. Enzinger FM. Epitheloid sarcoma. A sarcoma simulating a granuloma or a carcinoma. Cancer 1970;26:1029-41.

2. Chase DR, Enzinger FM. Epithelioid sarcoma. Diagnosis, prognostic indicators, and treatment. Am J Surg Pathol 1985;9:241-63.

3. Guillou L, Wadden C, Coindre JM, Krausz T, Fletcher CD. "Proximal-type" epithelioid sarcoma, a distinctive aggressive neoplasm showing rhabdoid features. Clinicopathologic, immunohistochemical, and ultrastructural study of a series. Am J Surg Pathol 1997;21:130-46.

4. Fisher C. Epithelioid sarcoma: The spectrum of ultrastructural differentiation in seven immunohistochemically defined cases. Hum Pathol 1988;19:265-75.

5. Spillane AJ, Thomas JM, Fisher C. Epithelioid sarcoma: The clinicopathological complexities of this rare soft tissue sarcoma. Ann Surg Oncol 2000;7:218-25.

6. Callister MD, Ballo MT, Pisters PW, Patel SR, Feig BW, Pollock RE, et al. Epithelioid sarcoma: Results of conservative surgery and radiotherapy. Int $\mathrm{J}$ Radiat Oncol Biol Phys 2001;51:384-91.

7. de Visscher SA, van Ginkel RJ, Wobbes T, Veth RP, Ten Heuvel SE, Suurmeijer AJ, et al. Epithelioid sarcoma: Still an only surgically curable disease. Cancer 2006;107:606-12.

8. Jones RL, Constantinidou A, Olmos D, Thway K, Fisher C, Al-Muderis $\mathrm{O}$, et al. Role of palliative chemotherapy in advanced epithelioid sarcoma. Am J Clin Oncol 
2012;35:351-7.

9. Pink D, Richter S, Gerdes S, Andreou D, Tunn PU, Busemann C, et al. Gemcitabine and docetaxel for epithelioid sarcoma: Results from a retrospective, multi-institutional analysis. Oncology 2014;87:95-103.
10. Guillou L, Wadden C, Coindre JM, Krausz T, Fletcher CD "Proximal-type" epithelioid sarcoma, a distinctive aggressive neoplasm showing rhabdoid features. Clinicopathologic, immunohistochemical, and ultrastructural study of a series. Am J Surg Pathol 1997;21:130-46. 